\title{
Synchronous Orbital and Gastric Metastasis of Invasive Lobular Breast Carcinoma, Mimic Primary Gastric Carcinoma-Case Report
}

Young Jin Choi ${ }^{1^{*}}$, Young Duck Shin ${ }^{1}$, Young Jin Song, Ho Chang Lee ${ }^{2}$ and Seung-Myoung Son ${ }^{2}$

${ }^{1}$ Department of Surgery, Anesthesiology, Chungbuk National University College of Medicine, Cheongju, Korea

${ }^{2}$ Deaprtment of Pathology, Chungbuk National University College of Medicine, Cheongju, Korea

"Corresponding author: Choi YJ, Department of Surgery, Chungbuk National University College of Medicine, Seongbong-ro 410, Heungdeok-gu, Cheongju 361-763, Korea, Tel: +82-43-269-6459; Fax: +82-43-266-6037; E-mail: yj0139@naver.com

Rec date: February 11, 2016; Acc date: April 12, 2016; Pub date: April 19, 2016

Copyright: (c) 2016 Choi YJ, et al. This is an open-access article distributed under the terms of the Creative Commons Attribution License, which permits unrestricted use, distribution, and reproduction in any medium, provided the original author and source are credited.

\begin{abstract}
Breast carcinoma is the most common primary origin of orbital metastasis. Orbital metastasis occurs through hematogenous spread and predominantly involves the choroid. On the other hand, breast carcinoma metastasis to the stomach is relatively rare. Invasive lobular carcinoma has a high tendency to metastasis to the stomach than ductal carcinoma. We present a case of invasive lobular carcinoma of breast with synchronous presentation of orbital and gastric metastasis. Gastroscopy revealed diffuse schirrous mass with pyloric obstruction and the biopsy was poorly differentiated carcinoma, mimic primary gastric malignancy. But on immunohistochemistry, both orbital and gastric mass showed hormonal receptor positivity that is agreement with primary breast lesion. Clinical suspicion, repeated endoscopic procedures, and intimate histologic analysis including immunohistochemistry is necessary for appropriate diagnosis, which might help to avoid unnecessary surgical procedure owing to incorrect diagnosis.
\end{abstract}

Keywords: Breast neoplasms; Lobular carcinoma; Neoplasm metastasis; Stomach; Orbit

\section{Background}

Breast carcinoma is the most common malignancy in worldwide and also second leading malignancy in South Korean woman [1]. The survival rates have increased over the years, because loco-regional and adjuvant therapy including hormonal therapy, chemotherapy and targeted therapy has progressed.

But the prognoses of advanced and metastatic breast carcinoma have not been changed. The common site of metastasis from breast carcinoma is bone, lung and liver and metastasis to orbit and gastrointestinal (GI) tract is relatively rare.

But the most common primary malignancy metastasis to orbit is breast carcinoma and the detection rate has been increased due to development in imaging study [2]. Rather, metastasis to GI tract from breast carcinoma is relatively rare. Invasive lobular carcinoma (ILC) has a high tendency to metastasis to the stomach than ductal carcinoma [3].

Because gastric malignancy is prevalent in South Korea [1], it can be difficult to distinguish from primary gastric malignancy on clinical, endoscopic, radiologic and histopathological features and can lead to unnecessary operative procedures.

Here, we present a rare case of invasive lobular carcinoma of breast with coincidence presentation with orbital and gastric metastasis, mimic primary gastric malignancy.

And, we also review recent literature about the usefulness of immunohistochemical (IHC) analysis in distinguishing primary gastric malignancy and gastric metastasis from breast carcinoma.

\section{Case Presentation}

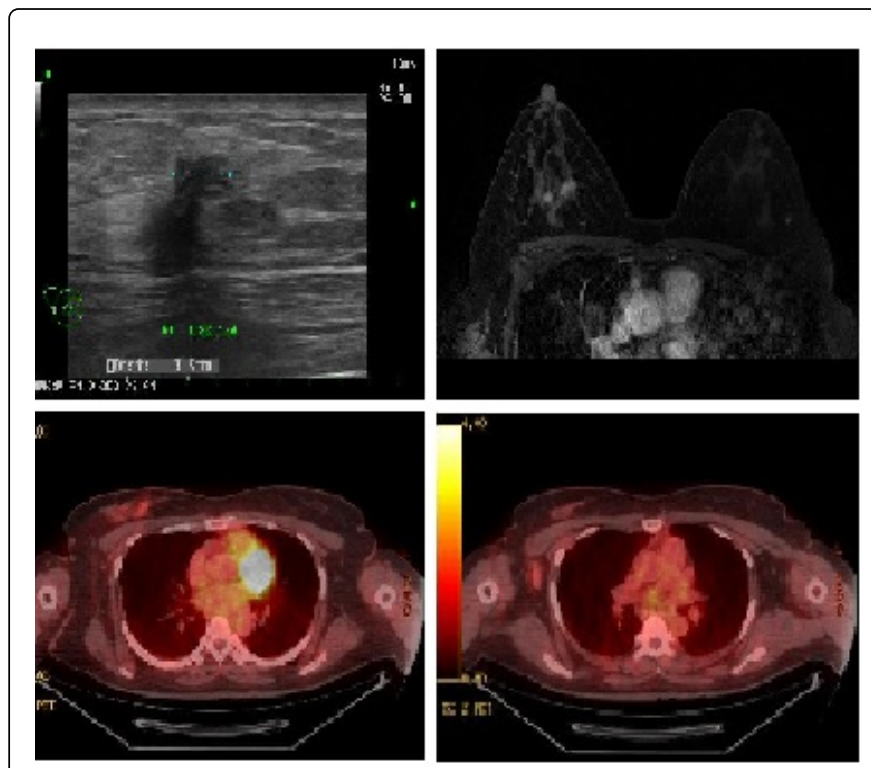

Figure 1: Images of Breast Ultrasonography, MRI and PET CT that shows two multiple irregular enhancing mass on right breast with axillary LN metastasis.

A 37-year-old woman visited with palpable right breast mass and left eyelid swelling developed 2 months before. She also had epigastric discomfort and there was history of repeated upper gastrointestinal (UGI) endoscopy 2 month before at local clinic and diagnosed as chronic gastritis on repeated endoscopic biopsy for pylorus thickening. 
Citation: Young Jin Choi, Young Duck Shin, Young Jin Song, Ho Chang Lee ,Seung-Myoung Son (2016) Synchronous Orbital and Gastric Metastasis of Invasive Lobular Breast Carcinoma, Mimic Primary Gastric Carcinoma-Case Report. Gen Med (Los Angeles) 4: 230. doi: $10.4172 / 2327-5146.1000230$

Page 2 of 5

A $1.5 \mathrm{~cm}$ sized irregular hard mass was palpated on right outer breast and multiple fixed conglomerated lymph nodes (LNs) were palpated on right axilla. Breast magnetic resonance imaging (MRI) and ultrasonography (USG) showed $1.5 \mathrm{~cm}$ and $1 \mathrm{~cm}$ sized multifocal malignant mass on right upper outer breast and multiple axillary lymphadenopathy.

Core needle biopsy results obtained from right breast mass was invasive lobular carcinoma. Laboratory analysis including liver function test, serum protein and creatinine was normal.

Hemoglobin was $12 \mathrm{~g} / \mathrm{dL}$ and CEA and CA15-3 were $4.36 \mathrm{ng} / \mathrm{mL}$ and $34.59 \mathrm{U} / \mathrm{mL}$, respectively. Abdomen pelvis USG showed negative finding except bilateral hydronephrosis. Positron emission tomography-computerized tomography (PET-CT) covering skull base to pelvic floor didn't show any distant metastasis except primary malignancy and metastatic axilla (Figure 1).

Orbital MRI showed infiltrating mass involving left extraconal and periorbital space and MRI guided incisional biopsy revealed metastatic adenocarcinoma from breast (Figures 2 and $5 a$ ).

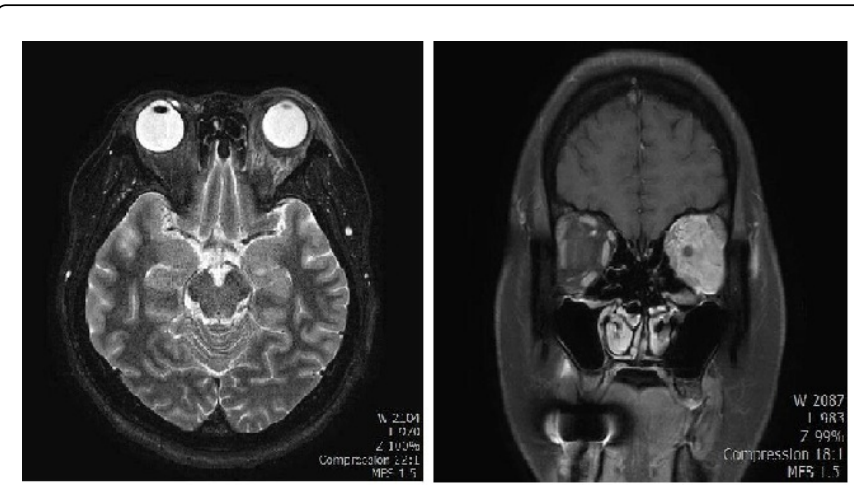

Figure 2: Orbital MRI showed diffuse abnormal enhancing infiltrated mass involving left extraconal and periorbital space.

For the purpose of palliation, she underwent right breast conserving surgery with level II axillary LN dissection. Postoperative histology showed completely excised $1.5 \mathrm{~cm}$ and $1 \mathrm{~cm}$ sized multifocal grade III invasive lobular carcinoma with clear resection margin and five out of 20 dissected LNs were positive of metastases.

IHC analysis revealed the cells were positive for both estrogen receptor (ER; 2+, 90\%) and progesterone receptor (PR; 2+, 60\%), and negative for E-cadherin and c-erb-B2 (Figure 3).

Additional IHC analysis showed positive for gross cystic disease fluid protein-15 (GCDFP-15) and negative for CDX-2, MUC-2, MUC-5.

About one month after surgery, she complained of recurrent epigastric fullness and pain. Repeated UGI endoscopy was preceded and there was diffuse schirrous mass on prepyloric antrum with pyloric obstruction.

Abdominal CT showed a gastric wall thickening around pylorus with impending pyloric obstruction without any obvious LN enlargement or metastatic spread; this was staged as T3 N0 M0 gastric malignancy (Figure 4).
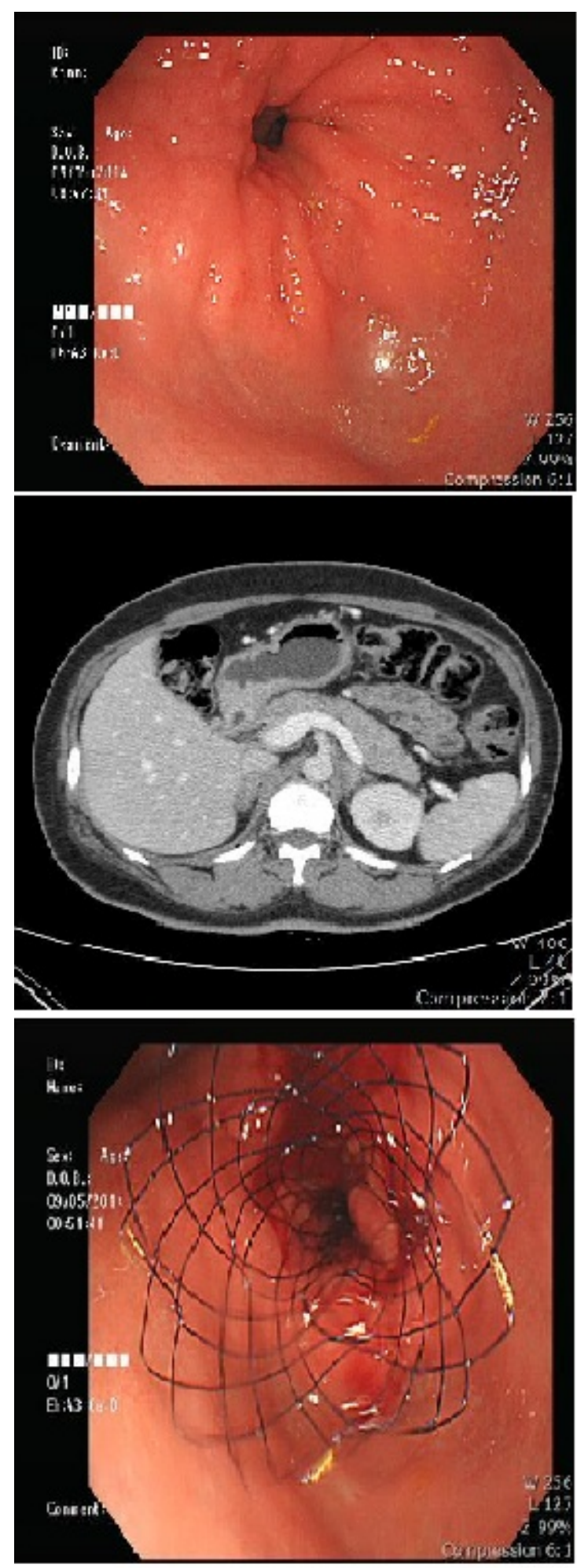

Figure 3: (3a) Diffuse schirrous mass on prepyloric antrum with pyloric obstruction was identified on gastroduodenoscopy. (3b) Abdomen CT with contrast demonstrating pyloric thickening. (3c) Duodenal stent was inserted to relieve pyloric obstruction. 
Citation: Young Jin Choi, Young Duck Shin, Young Jin Song, Ho Chang Lee ,Seung-Myoung Son (2016) Synchronous Orbital and Gastric Metastasis of Invasive Lobular Breast Carcinoma, Mimic Primary Gastric Carcinoma-Case Report. Gen Med (Los Angeles) 4: 230. doi: $10.4172 / 2327-5146.1000230$

Page 3 of 5

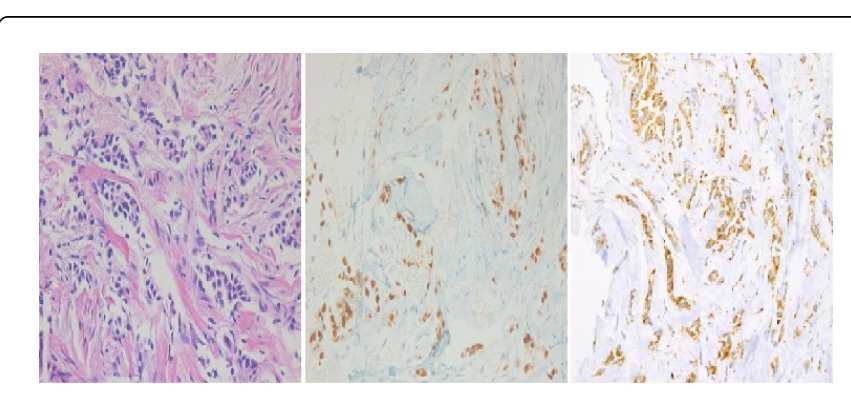

Figure 4: Histopathology of breast showed lobular carcinoma morphologically resembling signet ring cell carcinoma (4a). Hematoxylin and Eosin staining (x400), immunohistochemically positive for ER (4b) and negative for c-erbB2(4c).

Repeated endoscopic biopsy result was poorly differentiated carcinoma with atypical cells. Initially, gastric carcinoma was thought as synchronous primary malignancy. But on IHC, the cells were positive for ER (2+), PR (1+), GCDFP-15 and negative for CDX-2, cytokeratin (CK) 5, CK14, MUC-2, MUC-5, E-cadherin. These finding showed agreement with primary breast malignancy, consistent of metastatic carcinoma from the breast. Meanwhile, gastric malignancy showed c-erb-B2 overexpression, that was dissimilar finding with primary breast tumor (Figures $5 b-5 f$ ).

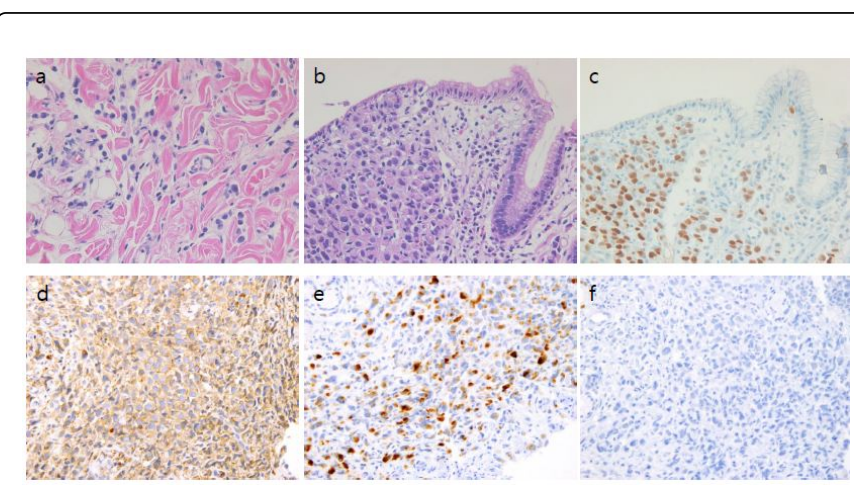

Figure 5: Histopathology of metastatic lesion. Orbital mass showed infiltration of neoplastic cells with histiocyte and monocytic appearance (5a), Hematoxylin and Eosin staining x400). Gastric biopsy showed poorly differentiated adenocarcinoma with signet ring cell morphology (5b), Hematoxylin and Eosin staining (x400), immunohistochemically positive for $\mathrm{ER}(5 \mathrm{c})$, c-erb B2(5d), GCDFP-15(5e) and negative for CDX-2(5f).

The patient did not have any family history of breast and gastric cancer. She did not have a prior history of lobular carcinoma in situ and had not been on hormonal replacement therapy.

After a multidisciplinary discussion, we have decided not to precede further surgical intervention for gastric lesion and the patient underwent pyloric stent insertion to symptomatic control. Systemic chemotherapy composed of paclitaxel $\left(80 \mathrm{mg} / \mathrm{m}^{2}\right)$ and trastuzumb $(2$ $\mathrm{mg} / \mathrm{kg}$ ) was delivered weekly. After 24 courses of chemotherapy, left orbital mass was decreased and foreign body sensation was improved. Because the eye symptom was improved, she did not proceed with orbital radiotherapy. Currently, since 18 months after initial treatment, she is under chemotherapy without change in regimen and orbital CT and endoscopic examination revealed that she has been showing partial response. She respond well to treatment with symptomatic improvement.

\section{Discussion}

About one-third of all orbital tumors are malignant and metastasis from breast carcinoma account for $28 \sim 58 \%$ of ocular and orbital metastasis. The orbital metastasis is detected as first sign of malignant disease or metastatic spread from breast carcinoma in about $10 \sim 30 \%$ of patients. Clinical presentation of orbital metastasis are diplopia, pain, blurred vision, exophthalmus, and a visible or palpable mass in the orbital or periorbital region, that was due to infiltration of cancer cell to extraocular muscle and orbital fat [4]. The histologic features of primary breast carcinoma can vary, and the pathologic findings of orbital metastases may also differ from the primary tumor. In this case, the main presentation of orbital metastasis was eyelid swelling and foreign body sensation and she didn't complain any other blurred vision or pain. Orbital metastasis represents hematogenous spread and the main treatment is palliative. The aim of treatment is improve quality of life and preserve visual activity. Main treatment option is radiotherapy and $60 \sim 80 \%$ of patient showed improvement in blurred vision. Generally, the patient with orbital metastasis have poor prognosis. In this case, initially, she had the plan to undergoing radiotherapy after palliative primary breast surgery [5].

Rather, gastric metastasis from breast carcinoma is relatively rare and the incidence has been estimated as $2-18 \%$. Most cases are diagnosed as one of the metastatic lesion out of multiple distant metastases. Clinical presentations include anorexia, early satiety, epigastric pain, bleeding and vomiting and in this case, she complained of dyspepsia and epigastric fullness in relation to the pyloric obstruction [6]. As seen in this case, metastatic gastric lesion show linitis plastica with diffuse infiltration of the muscularis propria and submucosa and rarely, external compression or discrete nodules may occur. Taal et al. [6] reported that $83 \%$ of patients with gastric metastasis had lobular histoloty as the primary breast pathology and the diffuse infiltration of stomach is the characteristic of metastasis from invasive lobular carcinoma of breast.

In this case, we thought the gastric lesion as primary gastric carcinoma. But clinical suspicion and endoscopic reevaluation, radiologic evaluation and discrete pathologic evaluation identified metastatic tumors and we can avoid unnecessary operative procedures. Macroscopic endoscopic evaluation is unhelpful in distinguishing primary and metastatic gastric malignancy. Endoscopic study may be normal in $50 \%$ of case, because gastric infiltration is frequently limited to the submucosa and seromuscular layer [7]. In this case, she diagnosed as chronic gastritis on previous repeated endoscopic biopsy, this may be originated from not doing deep submucosal biopsy. Deep and extensive biopsies and careful and comparative histologic analysis should be needed. However, lobular carcinoma of the breast may show signet ring cell morphology, this can be confused with a primary signet ring or diffuse-type gastric adenocarcinoma. Differentiation of a primary gastric poorly differentiated or signet ring cell carcinomas, from metastatic breast carcinomas may be difficult based on the histologic findings of the tumor. Diffuse type gastric adenocarcinomas and lobular carcinomas of the breast share a variety of cytomorphologic features, such as a single cell growth pattern and a signet ring cell appearance. However, primary and metastatic carcinomas of the GI tract have significantly different treatment and prognoses [8]. 
Citation: Young Jin Choi, Young Duck Shin, Young Jin Song, Ho Chang Lee ,Seung-Myoung Son (2016) Synchronous Orbital and Gastric Metastasis of Invasive Lobular Breast Carcinoma, Mimic Primary Gastric Carcinoma-Case Report. Gen Med (Los Angeles) 4: 230. doi: $10.4172 / 2327-5146.1000230$

Page 4 of 5

Detailed IHC study is the most helpful method in this case. Connel et al. [9] compared the IHC findings between primary gastric malignancy and metastatic gastric malignancy from breast carcinoma. Highly significant proportions of metastatic breast carcinomas were positive for ER (72\%), PR (33\%), GCDFP (78\%), and CK5/6 (61\%) compared with primary gastric carcinomas (ER, 0\%; PR, 0\%; GCDFP, $0 \%$; and CK5 $/ 6,14 \%$ ). Especially, ER, PR, and GCDFP were $100 \%$ specific. Meanwhile, there were no significant differences with any of the other immunostains (c-erb-B2, CK7, and MUC3) between the primary and metastasic lesions [10].

There were principal breast restricted markers currently in clinical use. ER expression can be powerful sensitive markers that can differentiate metastatic breast cancers. But it had low sensitivity $(\sim 50 \%)$ and specificity in metastatic lesions and furthermore, ER is expressed in a wide subset of non-breast carcinomas including endometrium and ovary. In contrast, ER expression is exceedingly rare in adenocarcinoma of GI tract, especially colorectal cancer. However, increased ER and PR have also been described in gastric adenocarcinoma in limited cases [11]. Gross cystic disease fluid is a pathological secretion from breast and GCDFP-15 expression is one of the breast specific markers with the range of sensitivity with $11 \%$ to $73 \%$ and specificity with $93 \%$ to $100 \%$. GCDFP-15 is not entirely breast restricted and expressed in apocrine gland origin malignancy including salivary glands, vulva, eyelids and tracheobronchial tree. Some gynecologic adenocarcinoma can also express GCDFP-15 (5 10\%), but the vast majority of GI tract are almost always negative. Mammoglobin and GATA expression are also breast specific markers which range of sensitivity with $26 \%$ to $84 \%$ and $32 \%$ to $95 \%$, respectively [12].

Conversely, CK 7 and CK 20 are useful cytokeratin markers for distinguishing carcinoma of metastatic and primary gastric lesion. CK $7+/$ CK20- phenotype is expressed in most breast, lung, and ovarian adenocarcinomas, whereas most intestinal adenocarcinomas are CK7-I CK20+. Additionally, homeobox protein CDX 2 which required for intestinal organogenesis and encoding nuclear transcription factors involved in the proliferation and differentiation of intestinal epithelium is expressed mainly in GI malignancy, 61\% of gastric cancers and $97 \%$ of colorectal cancers [13]. We didn't analyzed the expression of CK 7 and CK 20 in this patient, but positive expression of GCDFP-15, ER, PR, negative expression of CDX-2 is the critical finding for the diagnosis of metastatic breast carcinoma to stomach.

Curtit et al. [14] compared IHC findings between primary breast malignancy and metastatic lesion after treatment. Forty two patients showed discordance on IHC analysis, a switch in receptor status was verified for ER in $17 \%$ and PR in $29 \%$. In contrast, c-erb-B2 showed only $4 \%$ discordance rate. Chemotherapy, and especially anthracyclinebased chemotherapy, was associated with switch in ER status [14]. In this case, ER and PR showed positivity on metastatic gastric lesion that is agreement with primary breast disease; consistent with metastatic breast carcinoma. Rather, c-erb-B2 was expressed only in metastatic gastric lesion, this enables possibility of c-erb-B2 targeted therapy in addition to hormonal therapy and chemotherapy.

Hereditary gastric cancers account for 1-3\% of gastric cancer cases. Among them, hereditary diffuse gastric cancer (HDGC) is an inherited autosomal dominant syndrome, which caused mainly by E-cadherin gene $(\mathrm{CDH} 1)$ germline mutation. There is an association of HDGC with lobular breast cancer and the International Gastric Cancer Linkage Consortium (IGCLC) has established the clinical criteria for $\mathrm{CDH} 1$ genetic testing [15]. According to consensus guideline, the patient with diffuse gastric cancer with history of lobular breast cancer should be informed about genetic screening. But, present case concluded as primary lobular breast cancer with gastric metastasis and the patient did not have any family history of breast and gastric cancer. So, we did not proceed any further genetic testing.

\section{Conclusion}

The case of our patient showed synchronous presentation of orbital and gastric metastasis from invasive lobular breast carcinoma mimicking primary gastric malignancy. Clinical suspicion, repeated endoscopic procedures, and intimate histologic analysis including IHC are necessary for appropriate diagnosis and treatment, which might help to avoid unnecessary surgical procedure owing to incorrect diagnosis.

\section{References}

1. Jung KW, Won YJ, Kong HJ, Oh CM, Cho H, et al. (2015) Cancer statistics in Korea: incidence, mortality, survival, and prevalence in 2012. Cancer Res Treat 47: 127-141.

2. Raap M, Antonopoulos W, Dammrich M, Christgen H, Steinmann D, et al. (2015) High frequency of lobular breast cancer in distant metastases to the orbit. Cancer Med 4: 104-111.

3. Pectasides D, Psyrri A, Pliarchopoulou K, Floros T, Papaxoinis G, et al. (2009) Gastric metastases originating from breast cancer: report of 8 cases and review of the literature. Anticancer Res 29: 4759-4763.

4. $\quad \mathrm{Ng}$ E, Ilsen PF (2010) Orbital metastases. Optometry 81: 647-657.

5. Valenzuela AA, Archibald CW, Fleming B, Ong L, O'Donnell B, et al. (2009) Orbital metastasis: clinical features, management and outcome. Orbit 28: 153-159.

6. Taal BG, Peterse H, Boot H (2000) Clinical presentation, endoscopic features, and treatment of gastric metastases from breast carcinoma. Cancer 89: 2214-2221.

7. Qu Q, Zong Y, Fei XC, Chen XS, Xu C, et al. (2014) The importance of biopsy in clinically diagnosed metastatic lesions in patients with breast cancer. World J Surg Oncol 12: 93.

8. Jones GE, Strauss DC, Forshaw MJ, Deere H, Mahedeva U, et al. (2007) Breast cancer metastasis to the stomach may mimic primary gastric cancer: report of two cases and review of literature. World J Surg Oncol 5: 75.

9. Aurilio G, Disalvatore D, Pruneri G, Bagnardi V, Viale G, et al. (2014) A meta-analysis of oestrogen receptor, progesterone receptor and human epidermal growth factor receptor 2 discordance between primary breast cancer and metastases. Eur J Cancer 50: 277-289.

10. O'Connell FP, Wang HH, Odze RD (2005) Utility of immunohistochemistry in distinguishing primary adenocarcinomas from metastatic breast carcinomas in the gastrointestinal tract. Arch Pathol Lab Med 129: 338-347.

11. Kojima O, Takahashi T, Kawakami S, Uehara Y, Matsui M (1991) Localization of estrogen receptors in gastric cancer using immunohistochemical staining of monoclonal antibody. Cancer 67: 2401-2406.

12. Gown AM, Fulton RS, Kandalaft PL (2016) Markers of metastatic carcinoma of breast origin. Histopathology 68: 86-95.

13. Bayrak R, Haltas H, Yenidunya S (2012) The value of CDX2 and cytokeratins 7 and 20 expression in differentiating colorectal adenocarcinomas from extraintestinal gastrointestinal adenocarcinomas: cytokeratin 7-/20+ phenotype is more specific than CDX2 antibody. Diagn Pathol 7: 9.

14. Curtit E, Nerich V, Mansi L, Chaigneau L, Cals L, et al. (2013) Discordances in estrogen receptor status, progesterone receptor status, and HER2 status between primary breast cancer and metastasis. Oncologist 18: 667-674. 
Citation: Young Jin Choi, Young Duck Shin, Young Jin Song, Ho Chang Lee ,Seung-Myoung Son (2016) Synchronous Orbital and Gastric Metastasis of Invasive Lobular Breast Carcinoma, Mimic Primary Gastric Carcinoma-Case Report. Gen Med (Los Angeles) 4: 230. doi: $10.4172 / 2327-5146.1000230$

Page 5 of 5

15. Fitzgerald RC, Hardwick R, Huntsman D, Carneiro F, Guilford P, et al. (2009) Hereditary diffuse gastric cancer: updated consensus guidelines for clinical management and directions for future research. J Med Genet 47: 436-444. 